\title{
Dificuldades enfrentadas pelos enfermeiros na realização da classificação de risco no serviço de urgência e emergência
}

\author{
Difficulties faced by nurses in performing risk classification in the emergency and \\ emergency service
}

\begin{abstract}
Dificultades que enfrentan los enfermeros para realizar la clasificación de riesgos en el servicio de emergencia y emergencia
\end{abstract}

Cynthia Araújo Frota ${ }^{1 *}$, Sudário Vitor de Aquiar Lima², Amanda Oliveira Cardoso², Larissa Farias de Sousa ${ }^{2}$, Fernando Antonio da Silva Santos ${ }^{3}$, Maria Helena Alencar Trigo ${ }^{4}$, Ana Carolina Santos Cândido ${ }^{5}$, Simone dos Santos Rodrigues ${ }^{6}$, Maria Aparecida Pereira de Araujo ${ }^{6}$, Lucas Brito Peixoto ${ }^{7}$.

\begin{abstract}
RESUMO
Objetivo: Identificar na literatura as dificuldades enfrentadas pelos enfermeiros na realização da classificação de risco no serviço de urgência e emergência. Métodos: Realizou-se um estudo de revisão integrativa da literatura com busca de dados da Biblioteca Virtual em Saúde, LILACS, BVS e SCIELO tendo como descritores, acolhimento, enfermagem e emergência. Foram incluidos artigos que abordassem o tema, publicados em lingua inglesa e portuquesa no período dos anos de 2014 a 2018. Resultados: Foram identificados 152 artigos originais, dos quais selecionaram-se 10. Emergiram-se duas categorias temáticas: Acolhimento com classificação de risco e as Dificuldades dos Enfermeiros na realização da classificação de risco. Considerações Finais: Verificou-se que apesar do enfermeiro ser o responsável pela classificação de risco, este serviço apresenta várias dificuldades como à falta de capacitação dos enfermeiros para a realização da classificação de risco e aplicabilidade/avaliação do protocolo; déficit dos recursos materiais; discordância sobre a Classificação de Risco com o que é preconizado pela Política Nacional de Humanização.
\end{abstract}

Palavras-chave: Acolhimento, Enfermagem, Emergência.

\begin{abstract}
Objective: Identify in the literature the difficulties faced by nurses in the classification of risk in the emergency and emergency service. Methods: An integrative literature review study was carried out with the search for data from the Virtual Health Library, LILACS, BVS and SCIELO having as descriptors, reception, nursing and emergency. Included articles that addressed the theme, published in English and Portuguese in the period 2014-2018. Results: 152 original articles were identified between 2014 and 2018, of which 10 were selected. All material was analyzed using the Content Analysis technique. Two thematic categories emerged: Reception with risk classification and the Difficulties of Nurses in the accomplishment of the classification of risk. Final considerations: It was found that although the nurse is responsible for the classification of risk, this service presents several difficulties as the lack of training of nurses to carry out the risk classification and applicability / evaluation of the protocol; deficit of material resources; disagreement about the Risk Classification with what is advocated by the National Humanization Policy.
\end{abstract}

Keywords: Home, Nursing, Emergency.

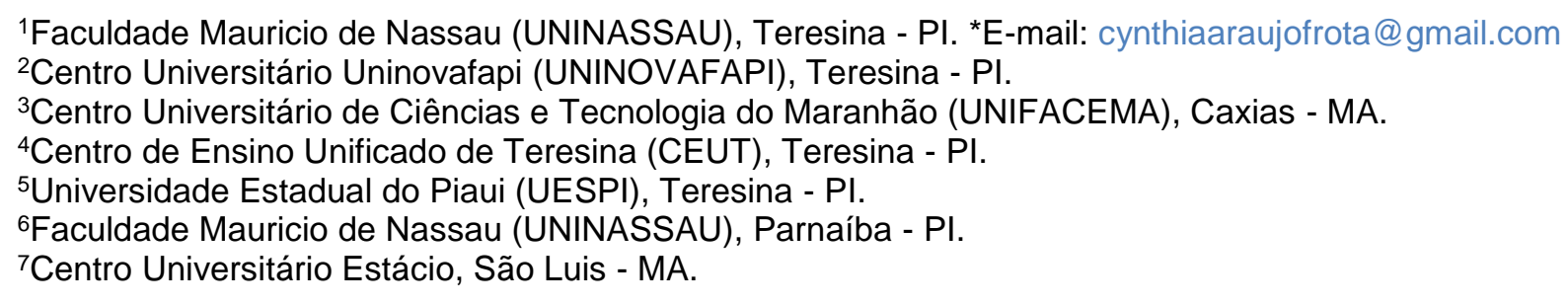




\section{RESUMEN}

Objetivo: Identificar en la literatura las dificultades enfrentadas por los enfermeros en la realización de la clasificación de riesgo en el servicio de urgencia y emergencia. Métodos: Se realizó un estudio de revisión integradora de la literatura con la búsqueda de datos de la Biblioteca Virtual en Salud, LILACS, BVS y SCIELO teniendo como descriptores, recepción, enfermería y emergencia. Se incluyeron artículos que abordaron el tema, publicados en inglés y portugués en el período 2014-2018. Resultados: Se identificaron 152 artículos originales disponibles entre los años 2014 a 2018, de los cuales se seleccionó 10. Todo el material fue analizado por medio de la técnica Análisis de Contenido. Se plantearon dos categorías temáticas: Acogida con clasificación de riesgo y las Dificultades de Enfermeros en la realización de la clasificación de riesgo. Consideraciones Finales: Se verificó que a pesar de que el enfermero era el responsable de la clasificación de riesgo, este servicio presenta varias dificultades como la falta de capacitación de los enfermeros para la realización de la clasificación de riesgo y aplicabilidad / evaluación del protocolo; déficit de los recursos materiales; discordia sobre la Clasificación de Riesgo con lo que es preconizado por la Política Nacional de Humanización.

Palabras clave: Recepción, Enfermería, Emergencia.

\section{INTRODUÇÃO}

Com a finalidade de gerenciar o acesso e fluxo de pacientes para priorização de atendimento nos serviços de saúde, o serviço de urgência e emergência passou adotar a Classificação de Risco (CR) como um processo dinâmico de identificação dos pacientes que necessitam de tratamento imediato, de acordo com o potencial de risco, agravos à saúde ou grau de sofrimentopara um atendimento de qualidade e com resolutividade em ampla escala, fazendo com que todos os pacientes sejam atendidos de forma satisfatória e em tempo hábil (SOUZA CC, et al., 2014).

A triagem nos serviços de Urgência e Emergência que classifica o risco de morte ao paciente, associado ao estado fisico em que ele busca os serviços de saúde, existe no Brasil desde o ano de 2002 mas em 2004 houve um acrescimento no seu desenvolvimento voltado para a humanização para melhor atender a população que busca os serviços de saúde incluindo os de urgência e emergência sendo assim a ser o acolhimento com classificação de risco, com ênfase na escuta adequada, criação de vínculos, garantindo um atendimento com resolutividade e priorizando os casos mais graves (INOUE KC, et al., 2015; PAIXÃO TCR, et al., 2015).

O serviço de urgência e emergência, inicialmente foi organizado tendo por objetivo de atender casos graves, de forma rápida e eficaz, prestando assistência em situações de emergência para usuários portadores de agravos agudos e casos agudizados de doença crônica, de traumas e doenças transmissíveis. Contudo, a disponibilização do atendimento imediato à população, com vistas à recuperação da saúde e à reversão de agravos crônicos, tem levado nas últimas décadas, ao aumento da demanda de pacientes, ocasionando superlotação nesses serviços (GANLEY L e GLOSTER AS, 2011).

Sequindo a CR, a assistência à pacientes em situações com risco iminente de morte não pode ser postergada e deve-se realizar o atendimento a esses pacientes de forma imediata, pois são situações que requerem emergência em atendiento médico. Pacientes que necessitam de atendimentos assistenciais à saúde que sejam menos grave, que não têm risco de morte iminente, mas que podem evoluir com complicações mais graves são atendindo em prazo de até uma hora, pois são classificados como situações de urgência.

E pacientes classificados como pouco urgente e que precisam ser atendidos no mesmo diapodém ter o atendimento realizado no prazo máximo de duas horas tendo avaliação médica de hora em hora até que seu quadro clínico esteja estável. E casos clínicos de menor complexidade classificados como não urgentes podém aguardar $\mathrm{o}$ atendimento com prazo de até três horas ou serem encaminhados para atendimento em unidade ambulatorialconvencional (OLIVEIRA GN, et al., 2011). 
Na explicação para o Acolhimento com Classificação de Risco (ACCR), o Ministério da Saúde determina que esse instrumento constitua uma diretriz operacional que uni as ações de acolhimento com as de classificação de risco do usuário. Isso significa que, no ACCR, o usuário que entra no Serviço Hospitalar de Emergência (SHE) é acolhido, ouvido, guiado à consulta de enfermagem, classificado de acordo com o grau de risco de seu agravo e atendido pelo médico segundo a urgência do caso (OLIVEIRA KKD, et al., 2013).

De acordo com Ministério da Saúde, humanização é compreendida como valorização do ser humano independente do meio sociocultural, economico, religioso, psicológico e fisico, tendo a inclusão desse indivíduo no processo de atendimento nos serviços de saúde de maneira que o mesmo se sinta acolhido de forma geral e tenha seu atendimento com resolutividade (BRASIL, 2014).

No Serviço Hospitalar de Emergência o aumento da demanda de pacientes que buscam por atendimento atinge diretamente a qualidade dos serviços oferecidos porque as abordagens dos profissionais se tornam focalizadas segundo a ordem de chegada do cliente e não de acordo com o seu grau de necessidade. Fatos como esse, revelam a necessidade de analisar questões que surgem a respeito do acesso pelos usuários a esses serviços (BELLUCCI JUNIOR JA e MATSUDA LM, 2012).

Assim, destaca-se que o acolhimento se trata de uma ação que possa ser desempenhada por qualquer profissional da área de saúde treinada para este fim. Contudo, a CR em específico, é responsabilidade do enfermeiro, que deve realizá-la através de consulta de enfermagem. O enfermeiro com base em um protocolo preestabelecido, classifica o usuário utilizando um sistema de cores discriminado em níveis de 5 cores, onde o vermelho significa (emergência), laranja (muita urgência), amarelo (urgência), verde (menor urgência) e azul (não urgência) (BELLUCCI JUNIOR JÁ e MATSUDA LM, 2012).

O enfermeiro interpreta os sinaisque o paciente demostra de forma ampla comtemplando os aspectos psicológicos, interpessoais, comunicativos e fisicocom cautela para que nenhuma informação passe despercebida, para verificar a credibilidade da informação clínica. Nesse sentido, o trabalho do enfermeiro na classificação de risco também é influenciado por aspectos sociais e pelo contexto de vida em que o usuário se encontra. Assim, o enfermeiro utiliza a avaliação intuitiva para exercer a classificação a partir da aparência física e do modo que o paciente apresenta o seu problema para um diagnostico preciso (ACOSTA AM, et., 2012).

Dessa forma, devido ao processo de tomada de decisão na CR acontecer em um cenário complexo e dinâmico, exige que decisões sejam aplicadas em um curto período de tempo, com informações limitadas, o que torna de fundamental importância da educação continuada para que o enfermeiro seja dotado de conhecimentos específicos para agir de forma eficaz diante de cada paciente que venha a procurer os serviços de saúde, além de experiência no atendimento a pacientes com diversas patologias, de modo a proporcionar uma prática segura e eficaz ao usuário (OLIVEIRA GN, et al., 2011).

A relevância do estudo consiste na visibilidade à atividade de classificação de risco desenvolvida por enfermeiros nas instituições hospitalares de urgência e emergência, no sentido de apontar as potencialidades e as dificuldades dessa prática do enfermeiro, presentes na realidade destes serviços. Diante ao apresentado, o estudo buscou identificar na literatura as dificuldades enfrentadas pelos enfermeiros na realização da CR no serviço de urgência e emergência.

Os estudos realizados enfatizam a avaliação dos beneficios positivos da CR e seus resultados nos serviços de urgência e emergência, juntamente com a utilização e validade do protocolo de Manchester, porem existem poucos estudos cientifícos elaborados com ponto central nas atividades realizadas pelos enfermeiros que trabalham na CR com destaques nas dificuldades, riscos e desafios encontrados por esses profissionais durante o trabalho desempenhado neste posto de trabalho.

\section{MÉTODOS}

Trata-se de uma Revisão Integrativa da literatura acerca das dificuldades enfrentadas pelos enfermeiros na realização da classificação de risco no serviço de urgência e emergência. Tendo como questão norteadora do estudo as dificuldades enfrentadas pelos enfermeiros durante a realização da CR aos pacientes que buscam os serviços de urgência e emergência. Apartir dessa quetão foram apontados como descritores de busca: Acolhimento AND Enfermagem AND Emergência. A busca dos dados foi realizada durante os meses de novembro de 2018 a janeiro de 2019. 
Figura 1 - Fluxograma representativo a respeito dos critérios de inclusão de exclusão.

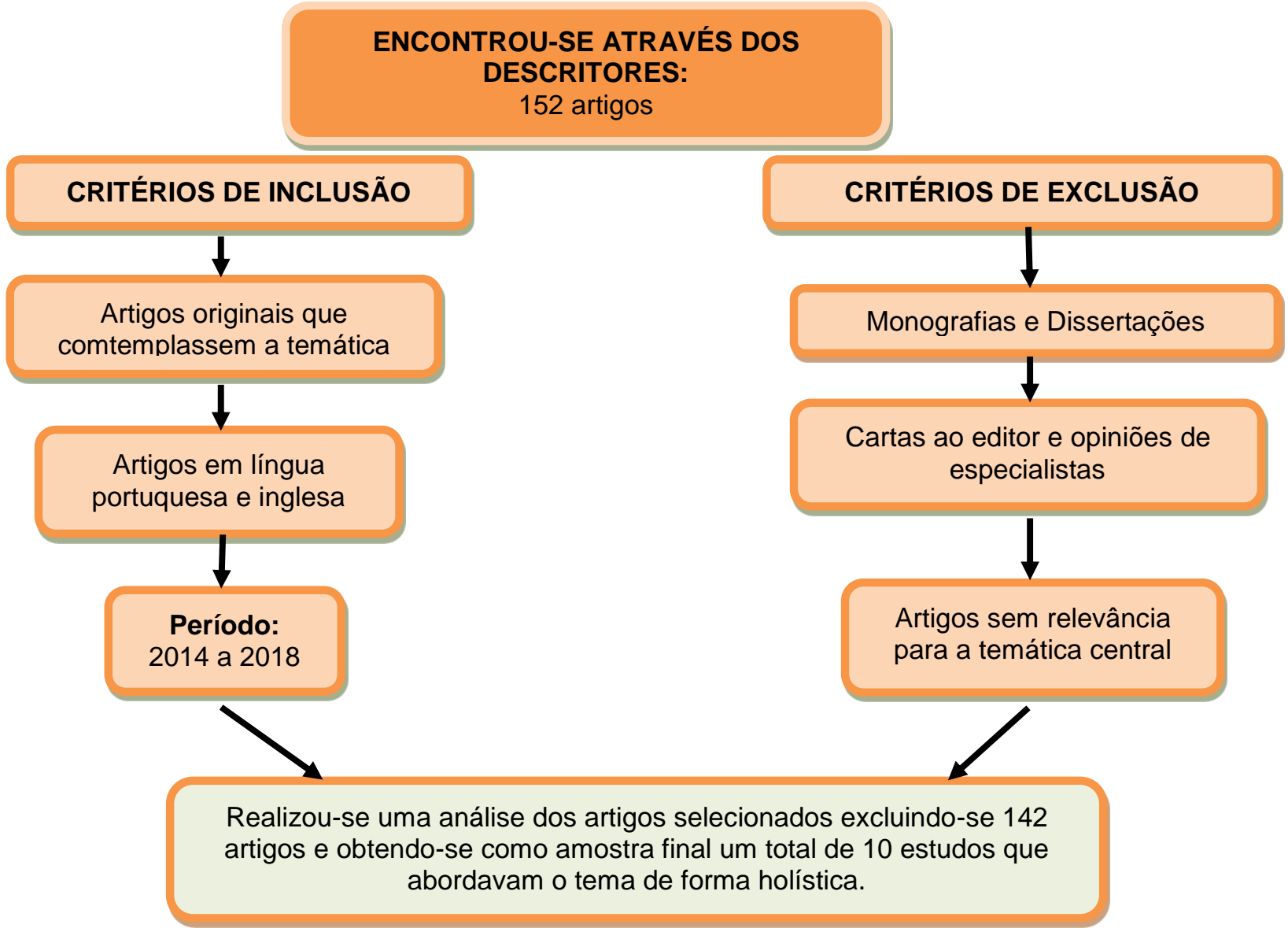

Fonte: Frota AC, et al., 2019.

Os estudos foram coletados nas bases de dados eletrônicas: Literatura Latino-Americana e do Caribe em Ciências da Saúde (LILACS), Base de dados de Enfermagem (BDENF) através da Biblioteca Virtual em Saúde (BVS) e na Biblioteca Eletrônica do Sientific Eletronic Library Online (SciELO).

A extração dos dados da amostra dos estudos primários selecionados foi executada por meio da utilização de um instrumento adaptado de coleta de dados, proposto e validado por pesquisadoras da enfermagem (URSI ES e GAVÃO CM, 2006). Tal instrumento adaptou-se a identificação do artigo, ano e local do estudo, características metodológicas da pesquisa, avaliação do rigor metodológico e nível de evidência. A análise dos dados foi realizada de forma descritiva. Para melhor compreensão dos achados e a discussão foi subdivida em categorias temáticas, o que exigiu a comparação dos estudos realizados com o conhecimento teórico.

\section{RESULTADOS}

A partir do estudo dos artigos estabeleceram-se variáveis relevantes para observação das produções científicas relacionadas à pesquisa. Os 10 artigos selecionados foram divulgados em periódicos nacionais. 0 período com maior número de publicações foi aquele compreendido entre 2015 a 2017 (08 artigos). Com relação à abordagem metodológica, a pesquisa qualitativa teve maior representatividade (4 artigos $=40 \%$ ), seguido da pesquisa quantitativa ( 3 artigos $=30 \%$ ). Os demais artigos são de corte retrospectivo $(10 \%)$, estudo de corte prospectivo (10\%) e um artigo de abordagem descritiva e analítica (10\%).

No que tange as bases de dados pesquisadas, o SciELO representou com 6 publicações (60\%) das publicações, seguida da LILACS com 3 publicações o que representou ( $30 \%$ das publicações), e por fim, a BDENF com 1 publicação, representando 10\% dos artigos selecionados. Os artigos estão sintetizados em ordem crescente conforme ao ano, que traz a distribuição das produções publicadas entre 2014 e 2018, segundo autor/ano, revista, base de dados e principais conclusões dos autores (Quadro 1). 
Quadro 1 - Distribuição dos artigos de acordo com os autores/ano, periódicos, base de dados e principais resultados.

\begin{tabular}{|c|c|c|c|}
\hline Autor/Ano & Periódico & Base de dados & Desfecho \\
\hline $\begin{array}{l}\text { SILVA MFN, et al., } \\
(2014) ;\end{array}$ & Rev. Latino-Am. Enfermagem & SciELO & $\begin{array}{l}\text { O fluxograma de atendimento mostrou-se de fácil aplicabilidade, sendo } \\
\text { facilitador para a busca, pela queixa, em cada prioridade de atendimento. }\end{array}$ \\
\hline $\begin{array}{l}\text { BECKER JB, et al., } \\
\text { (2015); }\end{array}$ & RevEscEnferm USP & SciELO & $\begin{array}{l}\text { Os resultados encontrados neste estudo demonstram que o protocolo } \\
\text { avaliado foi capaz de detectar pacientes com condições mais urgentes, bem } \\
\text { como identificar maior risco para internação hospitalar e óbito. }\end{array}$ \\
\hline $\begin{array}{l}\text { GUEDES HM, et al., } \\
\qquad(2015)\end{array}$ & Rev. Latino-Am. Enfermagem & SciELO & $\begin{array}{l}\text { Nas diversas cores do Sistema de Triagem Manchester, o óbito prevaleceu } \\
\text { nos pacientes que apresentaram a queixa de mal-estar no adulto, dispneia, } \\
\text { sofreram trauma craniano, trauma maior, diarreia e vômito. Quanto maior a } \\
\text { prioridade clínica maior a prevalência de óbito. }\end{array}$ \\
\hline $\begin{array}{l}\text { FARIAS JC, et al., } \\
\qquad(2016)\end{array}$ & Publ. UEPG Ci. Biol. Saúde & SciELO & $\begin{array}{l}\text { O reconhecimentodo acolhimento como elemento essencial no processo } \\
\text { de trabalhoda enfermagem é fundamental, pois a partir deste fazer é } \\
\text { possível promover umatendimento humanizado e resolutivo, estabelecido } \\
\text { pelo contato direto com o usuário. }\end{array}$ \\
\hline $\begin{array}{l}\text { NOVAES GPM, et al., } \\
\qquad(2016)\end{array}$ & Caderno Saúde e Desenvolvimento & LILACS & $\begin{array}{l}\text { A humanização é uma necessidade em todos os níveis da Saúde e tem } \\
\text { por objetivo ver o paciente como um todo, respeitando seus direitos e } \\
\text { autonomias. Dessa forma o que se observa é a contradição dessa } \\
\text { estruturação através do descaso, do mecanismo em ações dos } \\
\text { profissionais e autoritarismo. }\end{array}$ \\
\hline $\begin{array}{l}\text { PRUDÊNCIO CPG, et } \\
\text { al., (2016); }\end{array}$ & Revista Baiana de Enfermagem & LILACS & $\begin{array}{c}\text { Para a(o)s enfermeira(o)s, a classificação de risco é um mecanismo } \\
\text { indispensável para favorecer a otimização da assistência aos usuários que } \\
\text { apresentam quadros clínicos específicos de urgência e emergência. }\end{array}$ \\
\hline $\begin{array}{l}\text { SILVA PL, et al., } \\
(2016) ;\end{array}$ & RevEscEnferm USP & SciELO & $\begin{array}{l}\text { Houve um alto nível de satisfação do paciente, evidenciado pela forte } \\
\text { associação de usuários satisfação com os itens investigados. }\end{array}$ \\
\hline $\begin{array}{l}\text { HERMIDA PMV, et al., } \\
\qquad(2017) ;\end{array}$ & Revenferm UERJ & BDENF & $\begin{array}{l}\text { Os enfermeiros consideraram importante a CR na unidade de pronto } \\
\text { atendimento, contudo, encontram dificuldades quanto à unicidade de } \\
\text { conduta na sua implementação e registro, sinalizando a necessidade de } \\
\text { ações de educação permanente para uma melhor organização no } \\
\text { atendimento. }\end{array}$ \\
\hline $\begin{array}{l}\text { SPAGNUOLO RS, et } \\
\quad \text { al., (2017); }\end{array}$ & RevBrasPromSaúde & LILACS & \begin{tabular}{|c|} 
Os usuários possuem poucoconhecimento sobre a triagem com \\
classificação de risco, oque pode contribuir para a superlotação e prejudicar \\
o atendimento dos casos considerados urgentes.
\end{tabular} \\
\hline $\begin{array}{l}\text { MORENOECSG, et al., } \\
\text { (2018); }\end{array}$ & Revista Saúde e Pesquisa & SciELO & $\begin{array}{l}\text { Os usuários desconhecem o significado da CR na priorização dos } \\
\text { atendimentos à saúde. }\end{array}$ \\
\hline
\end{tabular}

Fonte: Frota CA, et al., 2019. 
Os artigos selecionados e analisados mostram que a realização da CR com o acolhimento humanizado nos serviços de saúde prestada pelos profissionais da saúde é de extrema importância para que haja um atendimento de excelência com um grau de resolutividade adequado, sendo também que os profissionais de enfermagem encontram dificuldades na realização da classificação de risco e a população tem pouco conhecimento dos critérios utilizados para avaliação na classificação de risco que muitas vezes se sentem insatisfeitos com o sistema de classificação. Foi emergido duas categorias temáticas para a discussão: Acolhimento com classificação de risco e Dificuldades dos Enfermeiros na realização da classificação de risco.

\section{DISCUSSÃO}

\section{Acolhimento com classificação de risco}

Dentre as prioridades de atendimento realizado no serviço de classificação de risco, o vermelho e o amarelo foram os que mais receberam sugestões de alterações após a avaliação, contudo a retirada destes itens que não fariam diferença na avaliação dos pacientes foi favorável, mantendo-se somente aqueles considerados de extrema importância. Dessa forma, a utilização do protocolo e do fluxograma, elaborados na pesquisa, mostrou-se de fácil aplicabilidade pelosenfermeiros, com resultados satisfatórios na $\mathrm{CR}$, o que facilitao processo de implantaçãodo atendimento a população (SILVA MFN et al., 2014).

O protocolo utilizado para $\mathrm{CR}$ é constituído por cinco níveis de classificações para a realização dos tendimento, sendo capaz de predizer diferentes desfechos, uma vez que, o grupo de alta prioridade estava associado a maiores taxas de internação hospitalar e óbitos. Sendo assim, as variáveis de idade, gênero e especialidades clínicas também apresentaram associação com maiores taxas de internação e óbito, dados que mostraram que os pacientes com maior idade do sexo masculino e pacientes atendidos em especialidades clínicas apresentaram maiores taxas de internação e óbito, principalmente aqueles classificados no grupo de maior prioridade (BECKER JB, et al., 2015).

Nos protocolos da CR apontados no estudo o sintoma mais evidenciado pelos pacientese que esteve presente em todas as $\mathrm{CR}$ foi a indisposição com incômodo físico sem queixas específicas relacionada a esse sintoma. Questiona-se a qualidade do atendimento com a realização da CR de forma fidedigna prestada a esses pacientes pelos profissionais de saúde no atendimento de urgência e emergência. Notou-se que para alguns profissionais de saúde seja mais conveniente e cômodo usar a nomeclatura de indisposição física para classificar um sintoma do paciente, pois o fluxograma relacionado a esse sintoma é bastante amplo (GUEDES HM, et al., 2015).

Destaca-se que no serviço de saúde é relevante que os profissionais busquem continuamente estratégiaspara promover um cuidado transcultural, integral e humanizado a população pois é através da utilização de tecnologias, como a do Acolhimento com Avaliação e CR, na rotina do serviço de urgência e emergência visando contribuir para uma qualidade na assistência ao indivíduo, famíliae comunidade, garantindo condições de vida e saúde a todos (FARIAS JC, et al., 2016).

Ressalta-se, que o campo de urgência e emergência deve ser pensado através de um grau de complexidade melhorando os recursos tecnológicos e a força de trabalho dos profissionais de saúde e suas equipes, atendendo ao paciente conforme sua necessidade específica e de maneira eficaz. Dessa forma, o Protocolo Brasileiro de Classificação de Risco é dividido em cinco discriminadores diferentes como requisitos para os atendimentos sendo eles: Área Vermelha, Área laranja, Área Amarela, Área Verde e Área Azul para avaliar e classificar os pacientes de acordo com a gravidade do seu estado de saúde (NOVAES GPM, et al., 2016).

Compreende-se que todos os instrumentos utilizados na busca de gerar uma melhoria na qualidade do atendimento são válidos, pois, auxiliam na tomada de decisão da(o) enfermeira(o) e buscam uma assistência uniformizada e ampla. Todavia, torna-se necessário considerar que todo paciente é único e a sua individualidade deve ser respeitada em todos os aspectos (PRUDÊNCIO CPG, et al., 2016). 
Avaliar é parte essencial no planejamento e na gestão do sistema de saúde, de forma que se busque a qualidade e resolubilidade em todos os casos. A CR nos serviços de urgência e emergência é uma atividade complexa e muito importante que depende tanto das habilidades e competências dos enfermeiros como também de fatores externos e subjetivos, como o ambiente de trabalho, recursos tecnologicos, relacionamento interpessoal e comunicação entre equips e pacientes (SILVA PL, et al., 2016).

A adoção de um sistema de CR no acolhimento aos pacientes, assegura o atendimento de acordo com a condição de saúde de cada paciente apresentada na avaliação inicial realizada pelo profissional enfermeiro na triagem. Nessa atividade, a triagem apresenta-se como um trabalho essencial para 0 correto redirecionamento dos pacientes, ao mesmo tempo em que tem como finalidade de descongestionar o serviço de urgência e emergência. No entanto, deve ser realizada por profissionais qualificados e de forma humanizada, aumentando a resolutividade dos problemas e a qualidade do atendimento para os pacientes (SPAGNUOLO RS, et al., 2017).

\section{Dificuldades dos Enfermeiros na realização da classificação de risco}

Identificou-se que é necessário ampliar a capacitação dos enfermeiros para a realização da $\mathrm{CR}$ e aplicabilidade/avaliação dos protocolos para melhorar a especificidade do mesmo, uma vez que, quanto maior a qualificação profissional e maior número de horas praticadas na classificação de risco, melhores serão os resultados na priorização do atendimento prestados aos usuários dos serviços de saúde de urgência e emergência (SILVA MFN, et al., 2014).

Houve limitações em estudos que estavam associadas a utilização de protocolo desenvolvidos localmente quanto à realização em centro único o que limitou a comparação com outros estudos, além disso, dificultou na generalização dos resultados para outras populações e regiões do país. Os resultados, porém, demonstram a importância da avaliação dos protocolos institucionais pelos profissionais habilitados que ainda não foram validados, além de poder ser um modelo a ser replicado (BECKER JB, et al., 2015).

Neste sentido, destaca-se quanto às desvantagens percebidas pelos enfermeiros no Acolhimento com Avaliação e CR, que a sobrecarga de trabalho ainda é vista como um fator negativo na implementação de novas práticas em saúde, devido a enfermagem ser sobrecarregada, porém, o enfermeiro tem grande importância no atendimento de urgência e emergência (FARIAS JC, et al., 2016).

Os fatores que interferem na assistência de enfermagem na CR são as tomadas de decisões corretas para identificar e diferenciar os pacientes que precisam de atendimento médico de urgência daqueles que podem aguardar, sendo um importante influenciador nos Serviços de Urgência e Emergência. Sendo assim, o enfermeiro precisa estar capacitado para classificar e se necessário reclassificar a prioridade dos atendimentos dos pacientes enquanto aguardam o atendimento (NOVAES GPM, et al., 2016).

Alguns fatores constituem-se em desafios, a exemplo do déficit em relação aos recursos materiais. $O$ prontuário eletrônico na unidade necessita do uso de computadores, mas esses equipamentos constantemente apresentam defeitos, favorecendo a superlotação da unidade. Em relação à verificação dos sinais vitais, especificamente a pressão arterial, também houve dificuldades, devido ao número de esfigmomanômetros serem insuficientes para a demanda de pacientes (PRUDÊNCIO CPG, et al., 2016).

Com propósito de amenizar as dificuldades do serviço de urgência, é de fundamental relevância conhecer a opinião e as expectativas das pessoas que recebem atendimento no serviço de acolhimento, uma vez que, isso seja um elemento importante na avaliação dos serviços de saúde.

Destaca-se, que o fato de cada enfermeiro avaliar e classificar de um jeito, constitue um fator negativo da CR merecendo cautela na sua interpretação e isso reforça que um importante componente do sistema de classificação é a avaliação da dor, elemento este que compreende aspectos subjetivos e complexos que envolvem tanto o paciente como o profissional. A avaliação apurada da dor pelo enfermeiro na CR fundamental para que o nível de prioridade do atendimento do paciente seja definido corretamente (HERMIDA PMV, et al., 2017). 
Foi evidenciado o desconhecimento do significado da avaliação na CR para priorização dos atendimentos à saúde por parte da população, bem como se percebeu o conformismo da maioria frente aos problemas funcionais que merecem adequação. Foi possível verificar ainda a existência a discordância sobre a CR com o que é preconizado pela Política Nacional de Humanização, visto que o profissional mais indicado para este atendimento é o enfermeiro e não o técnico de enfermagem (MORENO ECSG, et al., 2018).

È de grande importancia investigar e compreender os motivos que levam os usuários dos serviços de urgências e emergências hospitalares a ficarem descontentes e insatisfeitos com a realização da CR na triagem, por que pode haver alguma contraposição entre o que foi estabelecido na triagem pelo enfermeiro, o qual tem um conhecimento clínico baseado cientificamente, e o que o paciente e seus familiares acreditam que o paciente esteja classificado devido aos sintomas que o mesmo esteja manifestando (OLIVEIRA JLC, et al., 2017).

\section{CONSIDERAÇÕES FINAIS}

Verificou-se que a classificação de risco é indispensáve e sendo de grande importância para a organização na realização do atendimento aos pacientes com prioridade para casos clínicos graves sendo sua realização competência do enfermeiro. Algumas dificuldades nesse setor de atendimento foram evidenciadas como: a falta de capacitação dos enfermeiros para a realização da classificação de risco e aplicabilidade/avaliação dos protocolos institucionais; déficit dos recursos materiais; avaliação da dor de forma mais precisa; escuta dos usuários para obter um feedback do atendimento, bem como, a existência de discordância sobre a Classificação de Risco com o que é preconizado pela Política Nacional de Humanização. O enfermeiro deve possuir capacidade, agilidade, ética com o conhecimento clínico baseado na queixa principal, sinais vitais e sintomas, para identificar com certeza a classificação do usuário e o tempo previsto para o atendimento e se possível informar o usuário e familiares sobre o tempo provável de espera.

\section{REFERÊNCIAS}

1. ACOSTA AM, et al. Atividades do enfermeiro nos sistemas detriagem/classificação de risco nos serviços de urgência: Revisão integrativa. Revista Gaúcha de Enfermagem. 2012; 4(33):181-90.

2. BECKER JB, et al. Triagem no Serviço de Emergência: associação entreas suas categorias e os desfechos do paciente. RevEscEnferm USP. 2015; 49(5):783-789.

3. BELLUCCI JJ, Matsuda LM. Acolhimento com classificação de risco em serviçohospitalar de emergência: Avaliação da equipe de enfermagem. RevistaMineira de Enfermagem. 2012; 3(16):419-28.

4. BRASIL. Cadernos HumanizaSUS: Humanização do parto e do nascimento. Brasília (DF): Ministério da Saúde; 2014.

5. FARIAS JC, et al. Acolhimento com avaliação e classificação de risco comoferramenta de gestão: percepção dos enfermeiros. Publ. UEPG Ci. Biol. Saúde. 2016; 22(1):40-47.

6. GANLEY L, GLOSTER AS. Uma visão geral da triagem no departamento de emergência. Nurs Stand. 2011; 26(12):49-56.

7. GUEDES HM, et al. Relação entre queixas apresentadas por pacientes na urgência e o desfecho final. Rev. LatinoAm. Enfermagem. 2015; 23(4):587-94.

8. HERMIDA PMV, et al. Classificação de risco em unidade de pronto atendimento: discursos dos enfermeiros. Revenferm UERJ. 2017; 25:e19649.

9. INOUE, KC et al. Acolhimento com classificação de risco: avaliação da estrutura, processo e resultado. Revista Mineira de Enfermagem, 2015; 19(1): 13-28.

10. MENDES KDS, et al. Revisão integrativa: método de pesquisa para a incorporação de evidências na saúde e na enfermagem. Texto Contexto Enferm. 2008; 17(4): 758-64.

11. MORENO ECSG, et al. Acolhimento com Classificação de Risco em um Hospital da Rede Pública: Percepção dos Usuários. Revista Saúde e Pesquisa. 2018; 11(1):89-97.

12. NOVAES GPM, et al. Protocolos de classificação de riscoutilizados nas unidades de pronto atendimento (UPAS) 24 horas: uma questão dehumanização. Caderno Saúde e Desenvolvimento. 2016; 9(5):61-84.

13. OLIVEIRA GN, et al. Perfil da população atendida em uma unidade de emergência referenciada. Rev. Latino-Am. Enfermagem. 2011; 19(3).

14. OLIVEIRA JLC, et al. Acolhimento com classificação de risco: percepções de usuários de uma unidade de pronto atendimento. Texto \& Contexto-Enfermagem, 2017; 26.

15. OLIVEIRA KKD, et al. Impacto da implementação do acolhimento com classificação de riscopara o trabalho dos profissionais de uma unidade de pronto atendimento. Revista Mineira de Enfermagem. 2013; 1(17);148-56.

16. PAIXÃO TCR, et al. Nursing staff sizing in the emergency room of a university hospital. Rev Esc Enferm USPI, 2015 ;49(3):481-7.

17. PRUDÉNCIO CPG, et al. Percepção de enfermeira(o)s sobreacolhimento com classificação de riscono serviço de pronto atendimento. Revista Baiana de Enfermagem. 2016;30(2):1-10. 
18. SILVA MFN, et al. Protocolo de avaliação e classificação de risco de pacientes em unidade de emergência. Rev. Latino-Am. Enfermagem. 2014; 22(2):218-25.

19. SILVA PL, et al. Acolhimento com classificação de risco do serviço dePronto-Socorro Adulto: satisfação do usuário. Rev Esc Enferm USP. 2016; 50(3):427-433.

20. SOUZA CC, et al. Percepção do enfermeiro sobre a realização da classificação do risco no serviço de urgências. Invest. educ. enferm. 2014; 32(1): 78-86.

21. SPAGNUOLO RS, et al. Percepção dos usuários sobre a triagem com classificação de risco em um serviço de urgência de Cabo Verde. Rev Bras Promoç Saúde. 2017; 30(2): 249-254.

22. URSI ES, GAVÃO CM. Prevenção de lesões de pele no perioperatório: revisão integrativa da literatura. Rev Latinoam Enfermagem. 2006; 14(1):124-31. 